\title{
Skills training plus exposure therapy may reduce post traumatic stress in women who experienced childhood abuse
}

\author{
Cloitre M, Koenen K, Cohen L, Han H. Skills training in affective and interpersonal regulation followed by exposure: A \\ phase-based treatment for PTSD related to childhood abuse.J Consult Clin Psychology 2002 Oct; 70:1067-74.
}

\section{QUESTION: Does skills training followed by exposure therapy affect symptoms in women suffering post traumatic stress disorder related to childhood abuse?}

Design

Randomised trial with no allocation concealment.

Setting

New York; timeframe not specified.

\section{Participants}

Participants were 58 women with post traumatic stress disorder (PTSD) linked to physical or sexual abuse when they were under 18 years old (based on DSM-IV criteria). All were self referred via advertisements or word of mouth. Mean age 34 years; $46 \%$ Caucasian; 20\% African American; 15\% Hispanic; 19\% other ethnic groups. $48 \%$ had experienced both sexual and physical abuse, $39 \%$ had experienced sexual abuse only and 13\% had experienced physical abuse only. Exclusion criteria were age under 18 or over 65 years; organic or psychotic mental disorders; substance dependence; eating disorder; dissociative disorder; bipolar I disorder; borderline personality disorder, or suicide attempt or psychiatric hospitalisation within 3 months.

US National Institute of Mental Health.

For correspondence: M Cloitre, Child Study Center, New York

University School of Medicine, USA. marylene.cloitre@ med.nyu.edu

\section{Intervention}

Participants received 12 weeks of active treatment or wait list control. Active treatment consisted of 8 weekly sessions of skills training using the STAIR cognitivebehaviour programme followed by 8 sessions of prolonged exposure therapy using standard protocols. Women on the wait list were offered treatment at the end of the intervention period.

\section{Main outcome measures}

PTSD symptoms were measured using the Modified Post Traumatic Stress Disorder Symptom Scale. Capacity to regulate mood was measured using the General Expectancy for Negative Mood Regulation Scale. Anger problems and interpersonal and functional impairment were measured using the Anger Expression subscale and Inventory of Interpersonal Problems, respectively.

\section{Main results}

Compared to those on the wait list, women receiving skills training and exposure therapy had improved mood regulation, interpersonal skills and PTSD symptoms. Improvements were maintained at 3 and 9 month follow up.

\section{Conclusions}

Skills training combined with exposure therapy may have benefits for women suffering post traumatic stress disorder following childhood abuse.

\section{COMMENTARY}

This study marks a major advance in our understanding of how to treat PTSD, particularly as manifest in women who experienced abuse during childhood. Empirically validated treatments for child abuse may be more difficult to develop than treatments for survivors of adult traumas such as rape, because patients often present with a complex symptom picture that includes other serious problems in mood regulation and interpersonal disturbance. The reasons for seeking treatment may not be limited to intrusive memories of an event. Some clinicians are concerned that sufferers may have difficulty tolerating distress and managing anger and anxiety during exposure-based treatments. Focussing on specific traumatic memories or avoidance behaviours may not capture the full gamut of problems that these people experience. This concern is supported by the high dropout rate in exposure-based treatments compared to present-centred treatment or wait list conditions.

Cloitre $e$ al suggest that adult survivors of childhood abuse may tolerate exposure therapy and benefit more from this treatment than present-centred therapy as long as there is ample support and skills training. The authors recognise that day-to-day difficulties managing conflict and the power dynamics of relationships compel adult survivors of child abuse to seek treatment. Indeed, such problems in adulthood may reactivate earlier traumatic memories and lead to an exacerbation of PTSD. The STAIR programme provides patients with skills to help them modulate their emotions and confront interpersonal regulation. This may also help to establish a therapeutic alliance with the therapist. Both factors may help the survivor manage the distress associated with exposure, which in turn may reduce dropout.

This initial assessment of skills training plus exposure therapy found benefits in many symptom domains. To illustrate the necessity of the STAIR component, future research could directly test STAIR modified prolonged exposure against the STAIR and prolonged exposure components separately. It would be interesting to compare not only the efficacy of these treatment components, but also dropout rates. In the current study, it is not clear whether participants who dropped out did so during the STAIR or prolonged exposure components. Moreover, people with borderline personality disorder (BPD), current substance abuse, or subthreshold PTSD were excluded. Given the association between BPD and substance abuse with post traumatic stress syndrome and childhood trauma, it would be interesting to assess whether child abuse survivors with BPD respond to this intervention, and ask similar questions about the degree to which such patients require the skills training or exposure components of therapy.

In a research setting, investigators have the luxury of selecting participants on the basis of specified inclusion / exclusion criteria, designed to test study hypotheses. In clinical practice, however, therapists must often treat the very individuals who are excluded from clinical trials (due to not having the required number of symptoms, or having symptoms or conditions that render their conditions more complex, such as interpersonal or mood instability, suicidal ideation and 'character pathology,' for instance). Cloitre $e$ t al appear to have developed a treatment that is applicable to real life patients and clinical practice, but chose to empirically validate the treatment with a more traditional group of participants. It is encouraging that only 1 out of 31 participants in active treatment experienced a worsening of symptoms. This is important because, in practice, truly effective treatments are those that not only are safe for patients, but are also palatable to therapists. STAIR modified prolonged exposure provides a slightly longer timeframe in which to treat highly symptomatic patients and also provides the therapist with a method of preparing participants for such work. 